\title{
Quaderni
}

QUADERNI Communication, technologies, pouvoir

77 | Hiver 2011-2012

L'évaluation de la recherche

Du récit gaullien au storytelling franco-américain

Histoires d'une trajectoire

\section{Alexandre Eyries}

\section{(2) OpenEdition}

Journals

Édition électronique

URL : http://journals.openedition.org/quaderni/565

DOI : $10.4000 / q u a d e r n i .565$

ISSN : 2105-2956

Éditeur

Les éditions de la Maison des sciences de l'Homme

Édition imprimée

Date de publication : 5 janvier 2012

Pagination : 97-104

Référence électronique

Alexandre Eyries, « Du récit gaullien au storytelling franco-américain », Quaderni [En ligne], 77 | Hiver 2011-2012, mis en ligne le 05 janvier 2014, consulté le 06 mai 2019. URL : http://

journals.openedition.org/quaderni/565; DOI : 10.4000/quaderni.565 


\section{Politique}

du récit gaullien au storytelling franco-américain Histoires d'une trajectoire

Alexandre Eyries

Laboratoire I3M Université de Nice Sophia Antipolis
Cet article se donne pour mission d'analyser l'évolution du récit gaullien qu'a inventé André Malraux et dont il a été à la fois le principal héraut (héros) et le chroniqueur le plus fidèle et le plus attentif: « (...) la voix d'un homme d'exception, non inégal à son destin, la voix aussi d'un observateur passionné, d'un interlocuteur qui prête à ses partenaires ses propres accents sans trahir le plus souvent leur vérité profonde $»^{1}$.

André Malraux a raconté, en écrivain, l'histoire des derniers temps du mandat de son président de tutelle, il a amplifié et magnifié la légende du général de Gaulle. Comme l'écrit Lucien Sfez dans La symbolique politique, "le politique est affaire de symbolique. (...) Le politique est (...) affaire de légitimité, c'est-à-dire de croyances et de mémoires validées, en d'autres termes de symboles $»^{2}$. Ce dernier ne l'ignorait pas et entretenait sa légende par la scénarisation de ses conférences de presse et de ses allocutions télévisées.

Malraux a donné naissance à un mentir-vrai qui réinvente l'histoire et la vie politique, parce qu'il était conscient que le «pouvoir politique obtient finalement la subordination par le moyen de la théatralité. (...) Le pouvoir utilise d'ailleurs des moyens spectaculaires pour marquer sa prise en charge de l'histoire " ${ }^{3}$. En tant que témoin et acteur de l'histoire l'écrivain a raconté son général de Gaulle et son propre récit gaullien. Cet article se demandera si ce récit gaullien est resté identique ou s'il a connu de profondes mutations. Il essaiera de déterminer si ce récit s'est éloigné, avec l'accession de Nicolas Sarkozy au pouvoir, d'une certaine conception de la France, de sa grandeur et de son rôle à l'international. Pour Nicolas Sarkozy, Henri Guaino est l'homme 
chargé de l'écriture des discours présidentiels, le garant de la pérennité de la parole gaullienne et le porteur d'un véritable renouveau du discours politique français sous l'influence du storytelling ${ }^{4}$ et des spindoctors (d'experts de la communication) ayant conduit au succès Bill Clinton, Tony Blair et Barack Obama. Mon propos sera donc d'analyser les mutations profondes de la narrativisation de la vie politique française en partant des récits d'André Malraux jusqu'aux récits et discours écrits par Henri Guaino - en sa qualité de conseiller spécial - durant la campagne présidentielle de 2007 pour Nicolas Sarkozy qui, en tant que président de la République « est la figure (de la symbolique politique) $»^{5}$.

Mon article se fixera également pour but d'étudier l'influence du modèle américain dans la conception d'un discours politique fortement hybridé qui n'oublie pas ses origines gaulliennes mais tend de plus en plus vers une certaine américanisation du storytelling de la politique française.

\section{La politique française : un monde de narra- tions possibles?}

L'univers de la politique française, depuis la fin des années 1960, a évolué très rapidement et ce rythme s'est encore accéléré dans les dix dernières années entre l'accession au pouvoir de Nicolas Sarkozy le 6 mai 2007 et l'élection de Barack Obama à la tête des États-Unis en novembre 2008. Prenant l'exemple des narrations propres aux candidats américains à la présidence, Christian Salmon écrit, dans Storytelling: «les candidats à la présidence américaine ont tous en commun une histoire de mythes et de héros américains et ils doivent s'inscrire dans cette ligne narrative en créant, à partir de leurs origines familiales, leur propre histoire et en la confrontant avec celle des autres candidats pendant les campagnes électorales $»^{6}$. L'exemple narratif américain ayant fait florès en à peine trois ans dans la politique européenne, il convient d'interroger à présent les ramifications de ce phénomène dans les pratiques des hommes politiques français.

\section{André Malraux / Henri Guaino : un ou des récit(s) gaullien(s)?}

Entre l'André Malraux ministre du général de Gaulle (entre 1958 et 1969) et Henri Guaino, rien a priori de commun, si ce n'est un engagement en faveur du gaullisme, qu'il soit originaire ou tardif. Si, comme l'écrit Jacques Gerstlé dans un ouvrage publié en 1993, "la communication politique est l'ensemble des techniques et procédés dont disposent les acteurs politiques, le plus souvent des gouvernants, pour séduire, gérer et circonvenir l'opinion $»^{7}$, alors la problématique de la mise en récit de la vie politique française s'impose avec beaucoup de prégnance. La narrativisation du politique a été mise au service de la politique politicienne et de l'administration de la nation. Dans ces conditions, la communication politique « est utilisée pour interagir (...) persuader, convaincre, séduire, informer, commander, négocier, dominer $»^{8}$. Il est hors de doute que les textes de Malraux sur de Gaulle avaient une visée politique, et cherchaient à conforter l'aura symbolique du général, y compris au prix d'un mélange de fiction et de réalité: "les sentiments qui me lient au général de Gaulle étaient déjà anciens, bien que le récit traditionnel de notre première rencontre soit inventé : le général n'a certainement pas dit de moi, en Alsace, la phrase 
que Napoléon prononça sur Goethe, car, en Alsace, le colonel Berger n'a jamais été présenté au général de Gaulle »". Ce texte est révélateur d'un double niveau d'intrication de l'invention narrative et de la réalité historico-politique. En effet, le colonel Berger - nom de résistant de Malraux - est issu de l'un de ses romans. Il y a donc là à la fois une allusion intertextuelle à son œuvre et l'aveu d'une réinvention du récit politique. Malraux réécrit la scène de leur première rencontre et avoue à demi-mots qu'il aurait aimé que le général dise de lui : «Voilà un homme!», comme Napoléon l'a fait de Goethe. Le storytelling politique prépare l'élaboration d'un récit gaullien fondateur parce que fédérateur : "l'image symbolique, écrit Lucien Sfez, liaison fortifiée des contraires, pluralisme, mythe ${ }^{10}$. Mettant en récit le général, Malraux le pare du sens du dialogue et de l'écoute: "il établissait avec son interlocuteur un contact très fort, que l'éloignement semblait rendre inexplicable (...). Il y avait dans ce qu'il m'avait dit le poids que donne la responsabilité historique ${ }^{11}$. Le narrateur du récit gaullien fait de son personnage l'incarnation de la rencontre d'un homme et de son peuple. Cela tend à accréditer l'idée qu'une nation serait une puissance symbolique autant que militaire ou économique, ce que confirme Lucien Sfez dans La symbolique politique: «l'opération mythique du symbole a besoin d'un support de communication $»^{12}$. Dans son ouvrage Storytelling, Christian Salmon écrit : « en juillet 2007, le conseiller de Nicolas Sarkozy, Henri Guaino, résumait ainsi, dans un entretien au Monde, sa contribution à la campagne présidentielle : «la politique, c'est écrire une histoire partagée par ceux qui la font et ceux à qui elle est destinée. On ne transforme pas un pays sans être capable d'écrire et de raconter une histoire» ${ }^{13}$. Cette confession d'Henri Guaino est particulièrement éclairante car, à la volonté de transformation d'un pays, s'ajoute le désir de transformer un récit politique et national. La mise en récit de la vie du candidat à la présidentielle s'intègre dans le récit de la nation. L'hypotexte gaullien est infléchi et détourné par Henri Guaino «dont l'attachement au gaullisme a été complaisamment rapporté et mis en scène $»^{14}$. Le conseiller est lui-même un pur produit du storytelling car il a scénarisé sa dilection pour le gaullisme. Guaino s'attache à raconter une histoire des transformations futures de la France et n'a de cesse de se comporter en " barde de l'identité française $»^{15}$. Le storytelling donne ainsi naissance, au fil des déplacements, à une personnalisation du récit en fonction des objectifs visés. À Caen, les Normands sont «ces héros qui ont conquis l'Angleterre, Naples, la Sicile et Antioche, (qui ont) sillonné les mers et exploré le monde $»{ }^{16}$. Le candidat en campagne reprend la route et s'arrête en Bretagne où il célèbre la «grandeur » du marin : «Ce qu'il oppose à l'océan, ce n'est pas la force de ses bras, c'est la force de son esprit ${ }^{17}$. Le récit épouse les spécificités des régions sillonnées et frôle la démagogie en multipliant les apologies du terroir. Cette mise en récit de la campagne s'inscrit dans une logique de persuasion et de manipulation de l'électorat. Henri Guaino dans son jusqu'auboutisme apparaît comme un «Don Quichotte de la formule, harnaché de souvenirs, d'histoires et de légendes, chantant à chaque étape les héros fabuleux de sa chevalerie $»^{18}$. Assumant sa visée partisane, le conseiller spécial coud non pas de fil blanc mais de "clichés narratifs et (de) figures imposées (...) un récit scolaire, chantant la France historique et géographique ${ }^{19}$. 
Ce faisant, Henri Guaino révèle toute son talent de storyteller politique pour conjuguer conscience historique et visée prospective vers la «France d'après ». Au terme de cette sous-partie apparaît donc la transformation d'un récit gaullien fondateur en une multitude de récits déterminés par les situations qui les engendrent.

\section{D'" une certaine idée de la France "à des histoires de circonstance}

Les soixante dernières années ont vu la politique en France changer nettement de dimension grâce à l'accélération des flux d'information. La politique française s'est tournée vers l'efficacité et a recouru à des récits persuasifs. Une chose est certaine, l'ancienne obsession du général de Gaulle pour la défense de la souveraineté française s'est transformée pour suivre l'évolution de la société française et du monde. Dans ses Mémoires de guerre, le général écrit : «Toute ma vie, je me suis fait une certaine idée de la France... Le sentiment me l'inspire aussi bien que la raison. Ce qu'il y a, en moi, d'affectifimagine naturellement la France, telle la princesse des contes (...) vouée à une destinée éminente et exceptionnelle. J'ai, d'instinct, l'impression que la Providence l'a créée pour des succès achevés ou des malheurs exemplaires. (...) La France ne peut être la France sans la grandeur $»^{20}$. Cette citation prouve que de Gaulle est un écrivain et un styliste ayant lui-même contribué à sa propre mise en récit. Pour de Gaulle, la France est un personnage romanesque. Sa relation avec le pays qu'il a libéré et qu'il a gouverné est directement narrative : «Nous fûmes les personnages d'une curieuse intrigue, qu'il pressentit sans doute avant moi $\gg^{21}$. Le narrateur et le narré deviennent les personnages d'une mise en récit de la vie politique.

Christian Salmon cite une tribune intitulée Pour en finir avec un mythe et publié par Henri Guaino et Nicolas Sarkozy dans Le Monde : "pas plus qu'il ne suffit d'invoquer le nom du général de Gaulle pour incarner une certaine idée de la France, il ne suffit d'invoquer les grandes figures des luttes sociales pour incarner la justice, la solidarité ou la République $»^{22}$. Dès 2002, les deux hommes ont entrepris un travail de réécriture pour commencer un récit de conquête. Cette activité s'accompagne d'un travail de sape visant une certaine conception de la gauche dans une perspective clairement électoraliste : " il y a une mythologie de la gauche (...) qui est extrêmement efficace. (...) Cette gauche (...) qui se pare de vertus qu'elle n'a pas ne se contente pas de donner des leçons et de dénigrer ses adversaires. (...) Nous savons trop ce que nous devons aux valeurs de la République pour les laisser préempter par cette gauche imaginaire dont l'argumentaire se réduit aux procès d'intention ${ }^{23}$. Dès cette époque, une volonté de réécrire l'histoire politique contemporaine est à l'œuvre chez Henri Guaino et Nicolas Sarkozy. Ce désir de réécriture s'illustre dans des récits de circonstance n'ayant d'autre but que l'efficacité. Il y a une volonté de changer le monde en racontant une histoire. Stéréotypés et démagogiques ces récits en appellent aux affects des auditeurs. La Lorraine devient « cette terre sainte où c'est la même chose de prier Dieu ou de prier la France $»^{24}$. L'auteur des discours tisse une histoire de la «France de toujours, cette France qui est la vraie France, celle qui (...) est la somme de tous ces destins individuels, 
celle qui ne peut pas mourir parce que chacun d'entre vous veut qu'elle vive $»^{25}$. Partout les circonstances cachent le véritable but de ces discours de conquête. Ainsi, entre réécritures multiples et discours de circonstances, se dessine une tension, dans le paysage politique français vers une autre pratique discursive, nettement plus composite, de la politique.

\section{Vers une hybridation des discours politiques}

La constitution des discours politiques a radicalement changé et l'on assiste à un " emballement de la théâtrocratie au cours des dernières années. (...) La dramatisation médiatique destinée à une vaste audience tend à occuper la place qui était autrefois celle de la presse, de la littérature et du théâtre populaires ${ }^{26}$. Le storytelling transforme l'exercice de la politique en une immense foire aux vanités, mais aussi en une scène ouverte de théâtre qui entraine de nouveaux discours du récit politique.

\section{Une politique story américano-française}

Durant la campagne de 2007, une stratégie a été mise en place par l'équipe de Nicolas Sarkozy pour l'aider à parvenir à briguer la plus haute fonction, qui a été très inspirée par les techniques de communication mises au point par les équipes de Bill Clinton et de Tony Blair. Il ne s'agit donc pas «d'émouvoir ou de séduire, mais d'assigner à chacun sa place dans la superproduction «La France d'après», une distribution théâtrale pour quarante millions de figurants, un Puy-du-Fou géant, à l'échelle de la nation tout entière, convoquée pour jouer son rôle dans le péplum de la présidentielle $»^{27}$. Cette prégnance de l'imaginaire cinématographique américain dans la vie politique et dans sa mise en histoires n'est pas innocente. Le premier président américain à avoir utilisé en précurseur le storytelling a été Ronald Reagan qui, avant de faire de la politique, était acteur. Il a initié une nouvelle forme de gouvernance, faisant beaucoup de place aux anecdotes, que Christian Salmon appelle narrarchie. Voici l'une des anecdotes qu'a raconté un jour Ronald Reagan : "Vos vies nous rappellent qu'une de nos plus anciennes expressions reste toujours aussi nouvelle: tout est possible en Amérique si nous avons la foi, la volonté et le cour. L'histoire nous demande à nouveau d'être une force au service $d u$ bien sur cette planète $»^{28}$. Ce récit reaganien a profondément marqué l'imaginaire cinématographique hollywoodien au point que des années plus tard le film Independence Day de Roland Emmerich voit le personnage du président des États-Unis dire sensiblement la même chose.

Le storytelling accomplit un va-et-vient entre la politique et le cinéma, mais n'effectue qu'un aller simple entre les États-Unis et la France. Ce qu'ont bien compris les présidents américains, c'est qu'un récit bien maîtrisé est l'instrument d'une conquête réussie du pouvoir. L'équipe de campagne de Nicolas Sarkozy l'a doté d'un récit : «Il (s'agit) (...) de transformer le nouveau président et son entourage en personnages d'un «récit cohérent», de rendre populaire la saga de ses faits et gestes $»^{29}$. Dans ces conditions-là, tous les déplacements s'inscrivent dans un scénario bien huilé : «le pouvoir exécutif devient un pouvoir «d'exécution», de réalisation (au sens cinématographique) du scénario présidentiel considéré comme un enchaînement de décisions et qui fait 
l'objet d'un montage permanent, ce à quoi se résume l'activité hautement symbolique du pouvoir $\aleph^{30}$. Ce qui fait, par conséquent, de l'homme politique candidat à une élection un acteur.

\section{Deux acteurs de la comédie politique : Ségolène Royal et Nicolas Sarkozy}

La campagne présidentielle ayant abouti à l'élection de Nicolas Sarkozy a vu la candidate Ségolène Royal monter progressivement en puissance tout au long du premier tour des élections et même parvenir au second tour. L'opinion publique s'est prise de passion pour la story d'" une femme qui avait mis en échec le pouvoir patriarcal des 'éléphants du PS' $»^{31}$ et qui incarnait une femme libre, luttant contre le machisme en politique. Pour toutes ces raisons, sa mise en récit a recueilli un large succès. Lors du débat télévisé l'opposant à Nicolas Sarkozy, Ségolène Royal a bénéficié d'une «ferveur quasi religieuse entretenue autour de (sa) figure maternelle, (...) constamment en position d'écoute $\aleph^{32}$. Elle a manifesté sa compassion pour les personnes à forte résonance émotionnelle. En actrice aguerrie, Ségolène a tiré profit de l'exposition médiatique de $《$ son sourire $\|^{33}$. Elle a préparé avec soin le débat télévisuel qui l'opposait à Nicolas Sarkozy. Or, dès sa déclaration de candidature, celui-ci avait adapté les thèmes de la souffrance et de la rédemption à sa version du conservatisme compassionnel : «j'ai changé parce que les épreuves de la vie m'ont changé. (...) Je suis révolté par l'injustice et c'en est une lorsque la société ignore les victimes. Je veux parler pour elles, agir pour elles et même, s'il le faut, crier en leur nom $»^{34}$.

Lors du débat télévisé, Nicolas Sarkozy, en tant qu' " acteur (...) se soucie moins de contenu que de rythme, moins d'action que de mise en scène $\aleph^{35}$. En tant qu'acteur, le président se contente d'appliquer un scénario : «il raconte sans cesse des histoires peuplées de 'victimes méritantes' et de 'héros anonymes', de rencontres et de séparation, de succès et d'échec $»^{36}$. En tant que narrateur, il sait faire alterner les épisodes malheureux (le divorce) et les contes de fées (l'idylle avec Carla Bruni). Nicolas Sarkozy a compris qu'il devait scénariser son accession au pouvoir puis son exercice pour tenir en haleine l'opinion publique. Comme l'écrit Christian Salmon, l'exemple de Nicolas Sarkozy aura permis de mettre en évidence une profonde révolution narrative de la politique française : «le temps politique n'est plus scandé par les débats parlementaires, les Conseils des ministres, (...) il est l'objet d'une scénarisation permanente. On est insensiblement passé de la 'fonction' à la 'fiction' présidentielle $»^{37}$.

Au terme de cet article, il apparaît que la pratique du récit est indissociable de l'exercice de la politique et, ce, pour plusieurs raisons. Dans un premier temps, tout homme politique ou tout parti politique est porteur d'un projet idéologique, d'une vision du monde qui est mise en mots et en intrigue selon la définition qu'en donne Paul Ricœur à partir du terme grec muthos : "le muthos est l'agencement des faits en système (et) (ressortit à) l'art de 'composer les intrigues' $\|^{38}$. Le programme d'un candidat à une élection est un récit qui s'insère dans d'autres récits appartenant à l'histoire d'un pays. Ce récit emprunte à une mythologie spécifique que le candidat s'évertue à enrichir, à crédibiliser et à pérenniser: l'homme «politique nourrit ainsi la mythologie qui lui 
donne force, sens, et efficacité symbolique $»^{39}$. $\mathrm{S}$ 'agissant du récit gaullien et de son évolution, force $m$ 'est de constater que si le storytelling a fait autant florès ces dernières années en politique, c'est au nom d'une recherche constante de l'efficacité et d'une volonté farouche de contrôler l'opinion publique. Cette recherche pousse l'auteur des discours de Nicolas Sarkozy à recourir à de grosses ficelles narratives, à des citations convenues. Premier narrateur de ce que l'on a appelé par la suite le récit gaullien, André Malraux situe très justement la relation des Français à la politique de leur pays dans un entre-deux « entre la fiction et la satire, entre d'Artagnan et Croquebol $»^{40}$.

Malraux écrit ensuite que «Le héros de l'Histoire est le frère du héros de roman $»^{41}$, insistant ainsi sur le rapport romanesque à l'histoire, à son pays et à la politique de celui qui déclarait, dans un demi-sourire : " au fond, vous savez, mon seul rival international, c'est Tintin! Nous sommes les petits qui ne se laissent pas avoir par les grands. On ne s'en aperçoit pas, à cause de ma taille $»^{42}$.

Ainsi la politique constitue, sinon une histoire du monde, du moins un monde peuplé d'histoires !

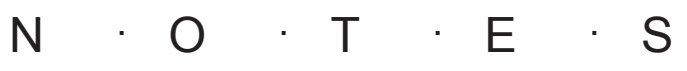

1. Marius-François Guyard, Antimémoires, notice dans André Malraux, Euvres complètes, tome III, Paris, Éditions Gallimard : collection Bibliothèque de la Pléiade, 1996, p. 1128.

2. Lucien Sfez, La symbolique politique, Paris, Presses Universitaires de France : collection «Que sais-je ?» n² 2400, 1988, p. 3.

3. Georges Balandier, Le pouvoir sur scènes, Paris, Éditions Balland : collection «Fondements», 1992, p. 21.

4. Christian Salmon définit le storytelling comme "l'art de raconter des histoires» dans Storytelling. La machine à fabriquer des histoires et à formater les esprits, Paris, Éditions de La Découverte [première édition 2007] : collection La Découverte / Poche, 2008, p. 7.

5. Lucien Sfez, La symbolique politique, op.cit., p. 12.

6. Christian Salmon, Storytelling. La machine à fabriquer des histoires et formater les esprits, Paris, Éditions de La Découverte : collection «La Découverte / Poche» [première édition 2007], 2008, p. 122.

7. Jacques Gerstlé, La communication politique, Paris, Presses Universitaires de France (PUF) : collection «Que sais-je ?» $\mathrm{n}^{\circ} 2652$ [première édition 1992], 1993, p. 4.

8. Ibid., p. 14.

9. André Malraux, Antimémoires, II, 1 dans Euvres complètes, tome III, Paris, Gallimard : collection «Bibliothèque de la Pléiade», 1996, p. 82.

10. Lucien Sfez, La symbolique politique, op.cit., p. 27.

11. Ibid., p. 98.

12. Lucien Sfez, op.cit.,p. 90. 
13. Christian Salmon, Storytelling. La machine à fabriquer des histoires et formater les esprits, op.cit., p. 200.

14. Ibid., p. 201

15. Ibid., p. 202.

16. Ibid., p. 202.

17. Ibid., p. 202.

18. Ibid., p. 202.

19. Ibid., p. 202.

20. Charles De Gaulle, Mémoires de guerre - L'Appel (1940-1942), Paris, Éditions Plon [première édition 1954], réédition Pocket, 1999 (nouvelle édition 2007), p. 7.

21. André Malraux, Antimémoires, II,3, op.cit., , p. 103.

22. Christian Salmon, Storytelling, op.cit., p. 201.

23. Henri Guaino et Nicolas Sarkozy, «Pour en finir avec un mythe» dans Le Monde du 11-04-2002, rubrique «Point de Vue».

24. Ibid., p. 203.

25. Ibid., p. 203.

26. Georges Balandier, Le pouvoir sur scènes, op.cit., pp. 9 et 11 .

27. Christian Salmon, Storytelling, op.cit., p 203-204.

28. Ronald Reagan cité par Christian Salmon dans Storytelling, op.cit., p. 125.

29. Christian Salmon, Storytelling, op.cit., p. 128.

30. Ibid., p. 128.

31. Ibid., p. 204.

32. Ibid., p. 207.

33. Ibid., p. 207.

34. Nicolas Sarkozy cité par Christian Salmon, Storytelling, op.cit., p. 206.

35. Christian Salmon, Storytelling, op.cit., pp. 215-216.

36. Ibid., p. 216.

37. Ibid., p. 216.

38. Paul Ricœur, Temps et récit 1. L'intrigue et le récit historique, Paris, Éditions du Seuil [première édition 1983] : collection Points Essais n² 227, 1991, p. 69.

39. Georges Balandier, Le pouvoir sur scènes, op.cit., p. 109.

40. André Malraux, Antimémoires, II, 3, op.cit., p. 111.

41. André Malraux, Les chênes qu'on abat... dans Cuvres complètes, tome III, Paris, Éditions Gallimard : collection «Bibliothèque de la Pléiade», 1996, p. 592.

42. Ibid., p. 591. 